\title{
The epsilon regime with twisted mass Wilson fermions
}

\author{
Oliver Bär, ${ }^{a}$ Silvia Necco ${ }^{b}$ and Andrea Shindler ${ }^{c}$ \\ ${ }^{a}$ Institut für Physik, Humboldt Universität zu Berlin, \\ Newtonstrasse 15, 12489 Berlin, Germany \\ ${ }^{b}$ CERN, Physics Departement, \\ 1211 Geneva 23, Switzerland \\ ${ }^{c}$ Instituto de Física Teórica UAM/CSIC, Universidad Autónoma de Madrid, \\ Cantoblanco E-28049 Madrid, Spain \\ E-mail: obaer@physik.hu-berlin.de, necco@mail.cern.ch, \\ andrea.shindler@uam.es
}

ABSTRACT: We investigate the leading lattice spacing effects in mesonic two-point correlators computed with twisted mass Wilson fermions in the epsilon-regime. By generalizing the procedure already introduced for the untwisted Wilson chiral effective theory, we extend the continuum chiral epsilon expansion to twisted mass WChPT. We define different regimes, depending on the relative power counting for the quark masses and the lattice spacing. We explicitly compute, for arbitrary twist angle, the leading $\mathrm{O}\left(a^{2}\right)$ corrections appearing at $\mathrm{NLO}$ in the so-called $\mathrm{GSM}^{*}$ regime. As in untwisted $\mathrm{WChPT}$, we find that in this situation the impact of explicit chiral symmetry breaking due to lattice artefacts is strongly suppressed. Of particular interest is the case of maximal twist, which corresponds to the setup usually adopted in lattice simulations with twisted mass Wilson fermions. The formulae we obtain can be matched to lattice data to extract physical low energy couplings, and to estimate systematic uncertainties coming from discretization errors.

KeYwords: Lattice QCD, Lattice Gauge Field Theories, Chiral Lagrangians, Lattice Quantum Field Theory

ARXIV EPRINT: 1002.1582 


\section{Contents}

1 Introduction 1

2 Chiral perturbation theory for twisted mass Wilson fermions 3

2.1 The chiral Lagrangian 3

2.2 Currents and densities 5

2.3 The PCAC mass and the twist angle 5

3 Effective theory in the epsilon regime $\quad 6$

$\begin{array}{ll}3.1 & \text { Epsilon expansion and power counting }\end{array}$

$\begin{array}{lll}3.2 & \text { Vacuum state and epsilon regime } & 8\end{array}$

$\begin{array}{lll}3.3 & \text { Epsilon expansion of correlation functions } & 9\end{array}$

4 Leading correction in the GSM* regime 10

$\begin{array}{lll}4.1 \text { Basic definitions } & 10\end{array}$

4.2 The PCAC mass in the GSM* regime 12

$\begin{array}{lll}4.3 & \text { Results } & 13\end{array}$

$\begin{array}{lll}\text { 4.3.1 Scalar and pseudoscalar correlators } & 13\end{array}$

$\begin{array}{ll}\text { 4.3.2 Axial and vector correlators } & 14\end{array}$

$\begin{array}{lll}4.4 & \text { Numerical estimates } & 15\end{array}$

5 Concluding remarks $\quad 16$

$\begin{array}{ll}\text { A Selected formulae and definitions for the epsilon regime } & 19\end{array}$

B Continuum correlators in the twisted basis $\quad 19$

B.1 Scalar and pseudoscalar correlators 20

$\begin{array}{lll}\text { B.2 Axial and vector correlators } & 20\end{array}$

$\begin{array}{ll}\text { C Notations for comparison with ref. [38] } & 21\end{array}$

$\begin{array}{ll}\text { D Example of group integrals with isospin breaking } & 22\end{array}$

\section{Introduction}

A precise matching of results obtained in lattice QCD with the predictions of the chiral effective theory is an important test of strong dynamics at low energies. In particular, it provides a way to check if chiral symmetry is spontaneously broken according to the expected pattern and eventually to extract from first principles the low-energy couplings which parametrize the effective theory.

With many recent calculations with $N_{f}=2,2+1$ getting close to the physical point (see the plenary talks $[1,2]$ presented at the 2009 lattice conference and references therein) this 
goal starts now to be realistic, although a reliable estimation of systematic uncertainties is still problematic. In particular, approaching the chiral limit in a finite box implies a detailed control over finite-size effects. The chiral effective theory provides information also on the volume dependence of physical observables: in the asymptotic region, where $M_{\pi} L \gg 1$, volume effects are expected to be exponentially suppressed. For practical purposes, for instance for the extraction of the pion decay constant, the empirical criterion $M_{\pi} L \gtrsim 4$ seems to be necessary in order to keep effects well below the statistical error typically present.

An alternative approach is to study QCD in a different kinematic corner, namely the $\epsilon$ regime [3, 4]: here finite-volume effects are polynomial instead of exponentially suppressed, and one can exploit the finite-size scaling properties of given observables in order to extract information about infinite-volume quantities. Since the chiral expansion obeys a different power counting with respect to the infinite volume, higher order corrections will be different: the matching of the chiral effective theory with lattice QCD will then provide low energy couplings which will be affected by different systematic uncertainties. A general agreement among those independent determinations is a good check on the validity of the approach.

Many quenched simulations in the $\epsilon$-regime have been performed [5-13], using Dirac operators which satisfy the Ginsparg-Wilson relation [14]. More recently, $\epsilon$-regime calculations with Ginsparg-Wilson fermions have been carried out also in the dynamical case, with $N_{f}=2[15-20]$ and $N_{f}=2+1[21,22]$. Since the Ginsparg-Wilson relation ensures exact chiral symmetry at finite lattice spacing [23], it guarantees many theoretical advantages, for instance the possibility to reach arbitrarily small quark masses and a continuum-like renormalization pattern. The price to pay is the high computational cost, which makes these simulations very challenging. In particular, approaching the continuum limit or exploring a broad range of physical volumes requires very big efforts.

Recently it has been realized that simulations in the $\epsilon$-regime are feasible also for Wilson-type fermions. In [24-26] first results obtained with Wilson twisted mass fermions have been presented. The use of a PHMC algorithm combined with an exact reweighting of a few low modes of the lattice operator turned out to be an important ingredient in this study. Analogously, in [27, 28] a reweighting algorithm has been proposed and successfully applied to simulate nHYP improved Wilson fermions in the $\epsilon$-regime (see also ref. [29]). In both cases continuum chiral perturbation theory (ChPT) describes the lattice data very well, although chiral symmetry is explicitly broken for Wilson-type fermions. Even though a scaling study would be necessary to systematically investigate lattice artifacts, this suggests that the impact of chiral symmetry breaking is mild and that it can be legitimate to match lattice results with the expressions of continuum ChPT.

We address this issue by means of Wilson Chiral perturbation theory (WChPT) [30, 31], which can be generalized to the twisted mass case [32-37]. In refs. [38, 39] we extended untwisted WChPT to the $\epsilon$-regime. ${ }^{1}$

The relevant issue is the relative power counting of the quark mass and the lattice

\footnotetext{
${ }^{1}$ Recently, WChPT in the $\epsilon$-regime has been adopted also to study the spectral density of the Wilson Dirac Operator at fixed topology [40].
} 
spacing (in units of $\Lambda_{\mathrm{QCD}}$ ). It turns out that for $m \sim a \Lambda_{\mathrm{QCD}}^{2}$ (GSM regime) the explicit breaking of chiral symmetry is still dominated by the quark mass, and lattice artifacts are highly suppressed. For mesonic two-point functions, the lattice spacing corrections start to appear at NNLO. On the other hand, if $m \sim a^{2} \Lambda_{\mathrm{QCD}}^{3}$ (Aoki regime), lattice artifacts compete with the quark mass, and corrections are substantial since they contribute already at LO.

In refs. $[38,39]$ we also introduced an intermediate regime $\left(\mathrm{GSM}^{*}\right)$, where discretization effects appear at NLO, and for this case we computed the leading corrections for several correlators. An important observation is that in this intermediate regime only one additional low energy coupling appears, namely $c_{2}$ [30]. In this paper we extend this study to the twisted mass case. While there will be many features in common to the untwisted case, some new aspects arise and will be discussed.

The paper is organized as follows: in section 2 we define the chiral Lagrangian of twisted $\mathrm{WChPT}$, currents and densities, and we recall the main related properties and definitions in an infinite volume; in section 3 we define the effective theory in the $\epsilon$-regime, we discuss the power counting and the rôle of the vacuum; in section 4 we compute leading corrections in the $\mathrm{GSM}^{*}$ regime for several 2-point correlators and we give numerical estimates for these corrections. Finally, we draw our conclusions in section 5 .

\section{Chiral perturbation theory for twisted mass Wilson fermions}

\subsection{The chiral Lagrangian}

Correlation functions computed with lattice simulations are affected by discretization errors, which can be analyzed using effective field theory. To obtain the correct form of the effective Lagrangian one proceeds in two steps [30]. First, one matches the lattice action used in the simulations with the appropriate Symanzik effective action. The Symanzik action is subsequently matched to a chiral Lagrangian which contains the standard continuum terms and appropriate additional operators that transform under chiral symmetry as the operators of the Symanzik effective theory. These additional operators describe the effects of the nonzero lattice spacing $a .^{2}$

In this paper we are interested in lattice actions with Wilson twisted mass fermions [43]. These actions are sometimes called Wilson-type fermions because they represent a simple generalization of the standard Wilson action. Their explicit form will not be needed in this paper (see ref. [44] for a review). We just recall that in our analysis we consider the lattice action with $N_{f}=2$ degenerate flavours, and bare mass parameters $m_{0}$ and $\mu_{\mathrm{q}}$. The untwisted quark mass $m_{\mathrm{R}}=Z_{m}\left(m_{0}-m_{\mathrm{cr}}\right)$ and the twisted quark mass $\mu_{\mathrm{R}}=Z_{\mathrm{P}}^{-1} \mu_{\mathrm{q}}$ are renormalized with renormalization factors computed in a mass independent scheme, and $m_{\mathrm{cr}}$ denotes the critical mass. Wilson twisted mass lattice QCD in the continuum limit is then equivalent to $\mathrm{QCD}[43]$ with a physical quark mass $m_{\mathrm{P}}=\sqrt{m_{\mathrm{R}}^{2}+\mu_{\mathrm{R}}^{2}}$. From now on we will drop the subscript $\mathrm{R}$ and all the quark masses are considered, unless specified differently, as renormalized in a mass independent scheme.

\footnotetext{
${ }^{2}$ For introductory lecture notes see ref. [41, 42].
} 
The chiral Lagrangian in twisted mass WChPT is essentially the same as for the untwisted case $[33,35-37,45]$. The only difference is a mass matrix that contains, besides an untwisted mass $m$, the twisted mass $\mu$.

In WChPT there are two sources of explicit chiral symmetry breaking, the quark masses $m, \mu$ and the lattice spacing $a$. The power counting is determined by the relative size of these parameters. The literature $[34,36]$ distinguishes two regimes with different power counting: (i) the generically small quark mass (GSM) regime where the quark mass is $\sim a \Lambda_{\mathrm{QCD}}^{2}$ and (ii) the Aoki regime where the quark mass is $\sim a^{2} \Lambda_{\mathrm{QCD}}^{3}$. Depending on the particular regime, the LO Lagrangian differs. Explicitly, in the GSM regime at leading order the chiral Lagrangian reads

$$
\mathcal{L}_{2}=\frac{F^{2}}{4} \operatorname{Tr}\left(\partial_{\mu} U \partial_{\mu} U^{\dagger}\right)-\frac{\Sigma}{2} \operatorname{Tr}\left(\mathcal{M}^{\dagger} U+U^{\dagger} \mathcal{M}\right)
$$

$F$ and $\Sigma$ are the familiar low energy couplings (LECs) appearing at LO in the continuum chiral Lagrangian and $U$ contains the pion fields in the usual way (see below). The mass matrix $\mathcal{M}$ is defined by ( $\sigma^{a}$ denotes the Pauli matrices)

$$
\mathcal{M}=\left(m \mathbb{I}+i \mu \sigma^{3}\right) .
$$

The field $U$ and the matrix $\mathcal{M}$ are written in the so called twisted basis. There is no $\mathrm{O}(a)$ contribution in the chiral Lagrangian $\mathcal{L}_{2}$, since we have absorbed it in the definition of $m$, which therefore represents the so-called shifted mass [30]. ${ }^{3}$

Eq. (2.2) can be used to define the polar mass $m_{\mathrm{P}}$ and a twist angle $\omega_{0}$ by

$$
\mathcal{M}=m_{\mathrm{P}} e^{i \omega_{0} \sigma^{3}}
$$

or, directly in terms of $m$ and $\mu$ :

$$
m_{\mathrm{P}}=\sqrt{m^{2}+\mu^{2}}, \quad \tan \omega_{0}=\frac{\mu}{m} .
$$

The subscript ' 0 ' serves as a reminder that the angle $\omega_{0}$ relates the two mass parameters in the chiral Lagrangian. ${ }^{4}$ We emphasize that $\omega_{0}$ is related to but not identical with the twist angle $\omega$ that we define in section 2.3.

We can easily go the so-called physical basis, where the quark mass matrix takes the standard form proportional to the identity, $\widetilde{\mathcal{M}}=m_{\mathrm{P}} \mathbb{I}$, by performing the following nonanomalous field transformation:

$$
U=W \widetilde{U} W \quad \text { with } \quad W=\exp \left(i \frac{\omega_{0} \sigma^{3}}{2}\right) .
$$

Here $\widetilde{U}$ denotes the field in the physical basis.

\footnotetext{
${ }^{3}$ The shifted mass is sometimes denoted by $m^{\prime}$. Here we drop the prime since we exclusively work with the shifted mass and we will never need the original mass parameter.

${ }^{4}$ Since we use the shifted mass to parametrize the chiral Lagrangian, the angle $\omega_{0}$ defined in (2.4) does not coincide with the one in ref. [35].
} 
If we move to the Aoki regime we have to add to the Lagrangian (2.1) the $\mathrm{O}\left(a^{2}\right)$ corrections $[46,47]$

$$
\delta \mathcal{L}_{a^{2}}=\frac{F^{2}}{16} c_{2} a^{2}\left(\operatorname{Tr}\left(U+U^{\dagger}\right)\right)^{2},
$$

since these contribute already at LO in this regime. Therefore, we expect the corrections due to a nonzero lattice spacing to be much more pronounced in the Aoki regime. Additional chiral logarithms proportional to $a^{2}$ appear in one-loop results of various observables like the pion mass and pion scattering lengths [47, 48]. Moreover, in infinite volume nontrivial phase transitions become relevant, and the sign of the LEC $c_{2}$ plays a decisive rôle for the phase diagram of the theory [30].

The NLO terms linear in $a$ can be found in refs. [31,36], but these terms will not be needed in the following.

\subsection{Currents and densities}

The currents and densities in the effective theory can be computed by adding appropriate source terms to the partition function of the theory and differentiating the resulting generating functional with respect to the sources [36, 49]. Alternatively, the currents and densities can be obtained using a standard spurion analysis, as described in [49]. Both methods have been discussed in detail in the literature. Here we simply summarize what is relevant for our computation with Wilson twisted mass fermions.

We assume the following definitions for the currents and densities at the quark level:

$$
\begin{aligned}
S^{0}(x) & =\bar{\psi}(x) \psi(x), & P^{a}(x) & =i \bar{\psi}(x) \gamma_{5} T^{a} \psi(x), \\
A_{\mu}^{a}(x) & =i \bar{\psi}(x) \gamma_{\mu} \gamma_{5} T^{a} \psi(x), & V_{\mu}^{a}(x) & =i \bar{\psi}(x) \gamma_{\mu} T^{a} \psi(x) .
\end{aligned}
$$

$T^{a}, a=1, \ldots, N_{f}^{2}-1$ are the Hermitean $\mathrm{SU}\left(N_{f}\right)$ generators satisfying the property $\operatorname{Tr}\left(T^{a} T^{b}\right)=\delta^{a b} / 2$. For $N_{f}=2, T^{a}=\sigma^{a} / 2$. The corresponding currents and densities at LO in the chiral effective theory read

$$
\begin{aligned}
\mathcal{S}^{0}(x) & =-\frac{\Sigma}{2} \operatorname{Tr}\left[U(x)+U^{\dagger}(x)\right], \\
\mathcal{P}^{a}(x) & =i \frac{\Sigma}{2} \operatorname{Tr}\left[T^{a}\left(U(x)-U^{\dagger}(x)\right)\right], \\
\mathcal{A}_{\mu}^{a}(x) & =i \frac{F^{2}}{2} \operatorname{Tr}\left[T^{a}\left(U(x)^{\dagger} \partial_{\mu} U(x)-U(x) \partial_{\mu} U^{\dagger}(x)\right)\right], \\
\mathcal{V}_{\mu}^{a}(x) & =-i \frac{F^{2}}{2} \operatorname{Tr}\left[T^{a}\left(U(x)^{\dagger} \partial_{\mu} U(x)+U(x) \partial_{\mu} U^{\dagger}(x)\right)\right] .
\end{aligned}
$$

These are the familiar expressions from LO continuum ChPT. The leading corrections of $\mathrm{O}(a)$ can be found in $[36,49]$. They are of higher order and we will not need them explicitly in the following.

\subsection{The PCAC mass and the twist angle}

There are various ways to define a twist angle. A popular way that has often been used is by the ratio

$$
\tan \omega=\frac{\mu}{m_{\mathrm{PCAC}}} .
$$


The denominator is given by the PCAC mass, which is an observable, instead of the shifted mass $m$. The former is defined as usual by

$$
m_{\mathrm{PCAC}}=\frac{\left\langle\partial_{\mu} A_{\mu}^{a}(x) P^{a}(y)\right\rangle}{2\left\langle P^{a}(x) P^{a}(y)\right\rangle} .
$$

In the classical continuum limit the PCAC mass is equal to $m$, and therefore $\omega=\omega_{0}$. For nonzero lattice spacings this is true only at LO, but violated at higher order, as we will derive in section 4.2. Hence, usually we have $\omega \neq \omega_{0}$.

Quite generally, $m_{\mathrm{PCAC}}$ is a function of $m, \mu$, the lattice spacing $a$ and the volume $V$. In principle this function can be "inverted" to obtain the shifted mass $m$ as function of the other parameters,

$$
m_{\mathrm{PCAC}}=m_{\mathrm{PCAC}}(m, \mu, a, V) \quad \Rightarrow \quad m=m\left(m_{\mathrm{PCAC}}, \mu, a, V\right) .
$$

In practice this relation can be computed perturbatively in the chiral expansion. Once it is known it allows us to express other observables as functions of the measurable quantity $m_{\text {PCAC }}$ instead of $m$. This will be the way we present our results in the latter sections.

A particularly interesting value for the twist angle is maximal twist, $\omega=\pi / 2$, where automatic $\mathrm{O}(a)$ improvement is guaranteed [50]. According to our definition (2.13) maximal twist is given by a vanishing PCAC mass.

\section{Effective theory in the epsilon regime}

\subsection{Epsilon expansion and power counting}

The discussion on the power counting in the previous section assumed an infinite volume. Finite-size effects due to a finite volume $V=T L^{3}$ with $L, T \gg 1 / \Lambda_{\mathrm{QCD}}$ can be systematically studied within ChPT $[3,4,51]$. If the pion Compton wavelength is much smaller than the size of the volume, $M_{\pi} L \gg 1$, finite-volume effects can be treated in the chiral effective theory by adopting the standard $p$-expansion, where the inverse box extensions are treated as small expansion parameters of the same order as the typical momenta: $1 / L, 1 / T \sim O(p)$. For asymptotically large volumes the finite-volume corrections are exponentially suppressed by factors $\exp \left(-M_{\pi} L\right)$.

On the other hand, for pion masses such that $M_{\pi} L \lesssim 1$ one probes the so-called $\epsilon$ regime of QCD [52-54]. In this regime the pion zero mode contribution $1 / M_{\pi}^{2} V$ to the pion propagator will eventually diverge in the chiral limit. Hence its contribution cannot be treated perturbatively but has to be computed exactly. This is achieved by a reordering of the chiral expansion by means of summing up all Feynman graphs with an arbitrary number of zero modes propagators. In the $\epsilon$-expansion one parametrizes the chiral field $U$ according to

$$
U(x)=U_{0} \exp \left(\frac{i}{F} \xi^{a}(x) \sigma^{a}\right)
$$


where the constant $U_{0} \in \mathrm{SU}\left(N_{f}\right)$ represents the collective zero-mode. The nonzero modes parametrized by $\xi^{a}$ are still treated perturbatively. These satisfy the condition

$$
\int_{V} d^{4} x \xi^{a}(x)=0
$$

since the constant mode has been separated. The $\epsilon$-expansion is now defined by using the counting rules

$$
M_{\pi}^{2} \sim \mathrm{O}\left(\epsilon^{4}\right), \quad 1 / L, 1 / T, \partial_{\mu} \sim \mathrm{O}(\epsilon), \quad \xi^{a} \sim \mathrm{O}(\epsilon) .
$$

With these counting rules the product $M_{\pi}^{2} V$ counts as $\epsilon^{0}$, just as its inverse. Consequently, all Feynman graphs that exclusively involve zero-mode propagators count as $\mathrm{O}(1)$ and are unsuppressed.

Once the counting for the pion mass is fixed, it determines the counting of the quark mass. In continuum ChPT the tree-level result $M_{\pi}^{2}=2 B m$, with $B=\Sigma / F^{2}$, fixes the counting $m \sim \mathrm{O}\left(\epsilon^{4}\right)$. The same line of argument has been applied to WChPT for untwisted masses $m$, and one obtains the same counting [38, 39]. Here we use it to deduce the counting for the polar mass $m_{\mathrm{P}}$ and the twisted mass $\mu$. The continuum tree-level pion mass for twisted mass QCD is $M_{\pi}^{2}=2 B m_{\mathrm{P}}$. Counting the pion mass squared as $O\left(\epsilon^{4}\right)$ we immediately find

$$
m_{\mathrm{P}} \sim O\left(\epsilon^{4}\right)
$$

This is the result that we would naively expect. With Wilson twisted mass fermions $m_{\mathrm{P}}$ plays the rôle of the physical quark mass, so the counting should be as for $m$ in the untwisted case. ${ }^{5}$ In terms of $m$ and $\mu$ this corresponds to have either both masses of $\mathrm{O}\left(\epsilon^{4}\right)$ or at least one of them of $\mathrm{O}\left(\epsilon^{4}\right)$ and the other of even higher order (in case one of the two masses is significantly smaller than the other).

In our computations we will keep both masses to be of $\mathrm{O}\left(\epsilon^{4}\right)$. This choice allows us to keep the computation general and our final formulæ, expressed in terms of rescaled masses $z_{m}=m \Sigma V$ and $z_{\mu}=\mu \Sigma V$, are valid for arbitrary twist angles. In particular, our final results will account for the special cases $\omega=\pi / 2$ (maximal twist) as well as for $\omega=0$, where we reproduce the results for standard Wilson fermions obtained in $[38,39]$.

The counting rule for the lattice spacing $a$ is now easily fixed. Quite generally, as in infinite volume, the power counting is determined by the relative size of $a$ and the quark mass. Since the counting of $m_{\mathrm{P}}$ is fixed we obtain the counting for $a$. The arguments are just as in WChPT with untwisted masses [38, 39], in particular, we carry over the definitions for three different regimes:

$$
\begin{aligned}
\text { GSM regime : } & a \sim \mathrm{O}\left(\epsilon^{4}\right), \\
\text { GSM }^{*} \text { regime : } & a \sim \mathrm{O}\left(\epsilon^{3}\right), \\
\text { Aoki regime : } & a \sim \mathrm{O}\left(\epsilon^{2}\right) .
\end{aligned}
$$

\footnotetext{
${ }^{5}$ Notice that in the Aoki regime the situation can be more complicated, since the standard LO relation between the pion mass and the polar mass is modified by $\mathrm{O}\left(a^{2}\right)$ corrections.
} 
Depending on the relative size of $a$ and $m_{\mathrm{P}}$ one of these counting rules is applicable. The GSM and the Aoki regimes have also been introduced in infinite volume WChPT. The $\epsilon$-expansion allows the introduction of the intermediate $\mathrm{GSM}^{*}$ regime [39], which defines the "transition region" between the other two regimes [38].

In the $\epsilon$-regime the topological charge plays a relevant rôle [55] and predictions in sectors of fixed topology can be given in the chiral effective theory. For Wilson-like fermions there is no unambiguous definition of the topological charge because the space of lattice gauge fields is connected and hence a division into topological sectors is somehow arbitrary. It is maybe possible, using the small real part of the eigenvalues of the Wilson operator, to have an operative definition of topology which leads to the correct continuum limit. Nevertheless in this work we concentrate on correlators where all sectors have been summed up also because numerical simulations are up to now not done at fixed topological charge.

\subsection{Vacuum state and epsilon regime}

A slightly unusual feature in twisted mass $\mathrm{WChPT}$ is the non-trivial ground state $U_{V}$, that is determined by a gap equation [35,37]. $U_{V}$ depends on the parameters $m, \mu$ and $a$, and this dependence affects observables when computed perturbatively. The reason is that $U_{V}$ enters the calculation if we compute correlation functions perturbatively by a saddle point expansion of the path integral around $U_{V}$. In the following we want to argue that $U_{V}$ is no longer needed in $\epsilon$-regime calculations where one integrates exactly over the collective constant mode.

In chiral perturbation theory (continuum or on the lattice, with a twisted or untwisted mass) we are interested in correlation functions defined by a functional integral,

$$
\langle\mathcal{O}\rangle=\frac{1}{\mathcal{Z}} \int \mathcal{D}[U] \mathcal{O}[U] e^{-S_{\chi}[U]}
$$

where $S_{\chi}$ is the effective action and $\mathcal{O}$ an effective (local) operator at a given order. $\mathcal{D}[U]$ denotes the measure for the path integral that needs to be properly defined [56, 57].

Suppose the effective action assumes its minimum for the constant field configuration $U=U_{V}$ and the integrand in (3.6) is strongly peaked around it. In this case we perform the standard saddle point expansion around $U_{V}$. We expand the field $U$ by the familiar ansatz

$$
U(x)=U_{V} \exp \left(i \pi^{a}(x) \sigma^{a} / F\right),
$$

and the measure is given by the formal product measure

$$
\mathcal{D}[U]=\mathcal{D}[\pi]
$$

leading to standard Gaussian integrals involving the propagator for the pion fields.

Alternatively, we may parametrize the $U$ field by isolating the collective zero-mode field as it is done in $\epsilon$-regime calculations. In this case we write

$$
U(x)=U_{V} U_{0} \exp \left(i \xi^{a}(x) \sigma^{a} / F\right)
$$


where the integration over $U_{0}$ is done exactly. The measure for this parametrization then reads $[4,52]$

$$
\mathcal{D}[U]=\mathrm{d}\left[U_{0}\right] \mathcal{D}[\xi][1-B(\xi)] .
$$

The measure factorizes and the correction $[1-B(\xi)]$ can be exponentiated to give an effective action $S_{\text {meas }}[\xi]$. In appendix A we give the explicit expression of $B(\xi)$ at $O\left(\epsilon^{2}\right)$. The main point here is that the measure $\mathrm{d}\left[U_{0}\right]$ for the constant mode is the standard Haar measure over the group manifold $\mathrm{SU}\left(N_{f}\right)$. Since it is left-invariant we can use $\mathrm{d}\left[U_{0}\right]=\mathrm{d}\left[U_{V} U_{0}\right]$ in computing the path integral. This implies, since we integrate over all constant fields, that the particular field $U_{V}$ is irrelevant, and instead of (3.9) we can directly parametrize the fields according to

$$
U(x)=U_{0} \exp \left(i \xi^{a}(x) \sigma^{a} / F\right)
$$

just as one does in calculations with an untwisted mass term [38, 39]. To summarize: In contrast to twisted mass WChPT in infinite volume (or in the p-regime) the ground state and the gap equation do not play a special rôle in the epsilon regime.

\subsection{Epsilon expansion of correlation functions}

In the following sections we mainly use the notation of ref. [39]. In appendix $\mathrm{C}$ we briefly discuss how the final formulæ should be written using the notation of ref. [38].

The discussion of the $\epsilon$-expansion of correlation functions can be carried over from ref. $[38,39]$. It is based entirely on dimensional arguments and once $m$ is replaced by $m_{\mathrm{P}}$ the entire discussion holds true for the twisted mass case. For this reason we do not repeat the arguments here but simply summarize the main results.

Correlation functions $\left\langle O_{1}(x) O_{2}(y)\right\rangle=\left\langle O_{1} O_{2}\right\rangle$ (for notational simplicity we suppress the dependence on $x, y)$ in $\mathrm{WChPT}$ are written as the sum of the corresponding continuum correlator plus a correction stemming from the nonzero lattice spacing,

$$
\left\langle O_{1} O_{2}\right\rangle_{\mathrm{WChPT}}=\left\langle O_{1} O_{2}\right\rangle_{\mathrm{ct}}+\delta\left\langle O_{1} O_{2}\right\rangle .
$$

The correction $\delta\left\langle O_{1} O_{2}\right\rangle$ receives contributions from both the effective action and the effective operator.

This correction is proportional to powers of the lattice spacing. At which order it contributes depends on the regime, cf. eq. (3.5).

In the GSM regime the correction starts with $\epsilon^{4}$ higher than the continuum contribution. In other words, the lattice spacing first affects the correlators at NNLO. Working to NLO one can ignore the correction and the continuum results are the appropriate ones.

In the GSM* regime the lattice spacing corrections enter at NLO. However, at this order only the $\mathrm{O}\left(a^{2}\right)$ correction proportional to $c_{2}$ contributes. The corrections linear in $a$, stemming from the corrections in both the action and the effective operators, are suppressed by one more power of $\epsilon{ }^{6}$

\footnotetext{
${ }^{6}$ These corrections of $\mathrm{O}(\mathrm{am})$ for Wilson fermions have been computed in ref. [38] for the pseudoscalar and scalar two point functions.
} 
Most pronounced are the corrections in the Aoki regime, where they contribute already to LO. In addition, the $\mathrm{O}\left(a^{2}\right)$ correction in the chiral Lagrangian cannot be completely expanded, it provides a zero-mode contribution of order $\epsilon^{0}$ that has to be treated exactly. As a result, the integrals over the constant mode are no longer the standard Bessel functions that one usually encounters in $\epsilon$-regime calculations.

Notwithstanding the complications in the Aoki regime, the main conclusion one can draw is that the lattice spacing corrections are typically suppressed, in the GSM regime to NNLO. This suppression of the lattice spacing corrections is one of the main reasons for the belief that Wilson fermions (twisted or not) are still a good choice for $\epsilon$-regime simulations despite their explicit chiral symmetry breaking.

\section{Leading correction in the GSM* regime}

\subsection{Basic definitions}

Lattice spacing corrections to correlation functions enter at NLO in the $\mathrm{GSM}^{*}$ regime. As mentioned in the previous section, only the $\mathrm{O}\left(a^{2}\right)$ term in the effective action contributes, and the NLO correction explicitly reads

$$
\left.\delta\left\langle O_{1}(x) O_{2}(y)\right\rangle\right|_{\mathrm{GSM}^{*}, \mathrm{NLO}}=-\left\langle O_{1, \mathrm{ct}}^{\mathrm{LO}}(x) O_{2, \mathrm{ct}}^{\mathrm{LO}}(y) \delta S_{a^{2}}\right\rangle+\left\langle O_{1, \mathrm{ct}}^{\mathrm{LO}}(x) O_{2, \mathrm{ct}}^{\mathrm{LO}}(y)\right\rangle\left\langle\delta S_{a^{2}}\right\rangle .
$$

The superscript "LO" in (4.1) refers to leading order in the $\epsilon$-expansion. In the twisted basis the $\mathrm{O}\left(a^{2}\right)$ term takes the same form as for standard Wilson fermions

$$
\delta S_{a^{2}}=\frac{\rho}{16}\left(\operatorname{Tr}\left(U_{0}+U_{0}^{\dagger}\right)\right)^{2},
$$

where we introduced the dimensionless quantity

$$
\rho=F^{2} c_{2} a^{2} V .
$$

The angled brackets in (4.1) stand for the functional integral over the non-constant fields $\xi^{a}(x)$ and the constant mode $U_{0}$. The integrals over the first ones are done perturbatively. This part is completely analogous to the untwisted case in ref. [38, 39], and we refer to appendix A for a collection of useful properties of the propagators.

The integral over the constant mode has to be done exactly, and here differences appear because of the twisted mass term $\mu$. The integrals we encounter in the twisted basis are of the type

$$
\left\langle g\left(U_{0}\right)\right\rangle=\frac{1}{Z_{0}} \int_{\mathrm{SU}(2)} \mathrm{d}\left[U_{0}\right] g\left(U_{0}\right) e^{\frac{z_{m}}{2} \operatorname{Tr}\left[U_{0}+U_{0}^{\dagger}\right]-i \frac{z \mu}{2} \operatorname{Tr}\left[\sigma^{3}\left(U_{0}-U_{0}^{\dagger}\right)\right]},
$$

where $Z_{0}$ is the partition function, obtained with $g\left(U_{0}\right) \equiv 1$. The parameters $z_{m}$ and $z_{\mu}$ are defined $a^{7}$

$$
z_{m}=m \Sigma V, \quad z_{\mu}=\mu \Sigma V .
$$

\footnotetext{
${ }^{7} z_{m}$ is the standard combination familiar from continuum ChPT, where it is usually denoted by $\mu$. In order to avoid confusion with the twisted mass we had to change this notation.
} 
For $z_{\mu}=0$ the integral (4.4) reduces to the standard one for an untwisted mass term. In this case it leads to expressions involving modified Bessel functions $I_{n}(x)$ with integer index $n$.

The same is true for $z_{\mu} \neq 0$, although the integral looks superficially rather different. This is immediately seen after performing a field redefinition to the physical basis. Performing the axial rotation (2.5) and using the invariance of the Haar measure, $\mathrm{d}\left[U_{0}\right]=\mathrm{d}\left[W U_{0} W\right]$, eq. (4.4) can be written as

$$
\left\langle g\left(U_{0}\right)\right\rangle=\frac{1}{Z_{0}} \int_{\mathrm{SU}(2)} \mathrm{d}\left[\widetilde{U_{0}}\right] g\left(W \widetilde{U_{0}} W\right) e^{\frac{z}{2} \operatorname{Tr}\left[\widetilde{U_{0}}+{\widetilde{U_{0}}}^{\dagger}\right]} \equiv\left\langle g\left(W \widetilde{U_{0}} W\right)\right\rangle_{\mathrm{phys}},
$$

where $z$ in the exponent is now given by

$$
z=m_{\mathrm{P}} \Sigma V=\sqrt{z_{m}^{2}+z_{\mu}^{2}} .
$$

The Boltzmann factor in the integrand assumes now the standard form with the polar mass $m_{\mathrm{P}}$ entering the exponent, hence the index 'phys' on the r.h.s of eq. (4.6). The representation (4.6) is particularly useful for doing actual calculations, since many results for integrals with untwisted masses can be taken over to the twisted mass case.

Note that twisted mass Wilson fermions break the $\mathrm{SU}(2)$ isospin symmetry to a residual $\mathrm{U}(1)$. This implies that completeness relations for $\mathrm{SU}\left(N_{f}\right)$ generators, which are repeatedly used in standard $\epsilon$-regime calculations [52], cannot be applied in our case. This poses a slight computational nuisance but no serious difficulty. In appendix D we give an example for the computation of zero mode integrals in presence of isospin breaking.

We calculated the correction (4.1) for a variety of mesonic correlation functions. For the presentation of our results we find it useful to introduce the following notation. First, translation invariance allows us to write

$$
\left\langle X^{a}(x) Y^{b}(y)\right\rangle=C_{X Y}^{a b}(x-y),
$$

where $X^{a}$ and $Y^{a}$ represent one of the densities or currents listed in (2.9)-(2.12). We suppress the spacetime index in the currents and only make the isospin index explicit. The vector and axial correlators we consider in section 4.3.2 refer to the temporal component of the currents.

In the $\mathrm{GSM}^{*}$ regime, $C_{X Y}^{a b}(x-y)$ can be written through NLO as the sum of the continuum correlator and a correction proportional to $a^{2}$,

$$
C_{X Y}^{a b}(x-y)=C_{X Y, \mathrm{ct}}^{a b}(x-y)+C_{X Y, a^{2}}^{a b}(x-y) .
$$

The continuum correlator (for generic $N_{f}$ ) at NLO can be found in the literature [52] (see also refs. [58] and [39]). Since we present our final results in the twisted basis, for the reader's convenience we collect a few relevant formulae and the expressions for the continuum parts of the correlation functions in the twisted basis in appendix B.

For the matching with numerical results obtained in lattice simulations one is often interested in the correlation function integrated over the spatial components,

$$
C_{X Y}^{a b}(t)=\left.\int d^{3} \vec{x} C_{X Y}^{a b}(x-y)\right|_{y=0}=C_{X Y, \mathrm{ct}}^{a b}(t)+C_{X Y, a^{2}}^{a b},
$$


To the order we are working here the correction $C_{X Y, a^{2}}^{a b}$ is independent of $t$ and only shifts the constant part of the continuum result.

\subsection{The PCAC mass in the GSM* regime}

The first observable we compute is the PCAC mass defined in (2.14). This allows us to express the results for other correlators as a function of $m_{\text {PCAC }}$ instead of $m$ (or, equivalently, as a function of $\omega$ instead of $\left.\omega_{0}\right)$.

In the chiral effective theory we write the numerator in (2.14) as (no summation over the flavor index $a$, with $a$ restricted to 1,2 )

$$
\begin{aligned}
\left\langle\partial_{\mu} \mathcal{A}_{\mu}^{a}(x) \mathcal{P}^{a}(0)\right\rangle & =C_{\partial A P}(x), \\
C_{\partial A P}(x) & =C_{\partial A P, \mathrm{ct}}(x)+C_{\partial A P, a^{2}}(x),
\end{aligned}
$$

and similarly for $\left\langle\mathcal{P}^{a}(x) \mathcal{P}^{a}(0)\right\rangle$. To leading order in the $\epsilon$-expansion we find

$$
\begin{aligned}
C_{\partial A P, \mathrm{ct}}(x) & =\frac{\Sigma}{V} \frac{I_{2}(2 z)}{I_{1}(2 z)} \cos \omega_{0}, \\
C_{P P, \mathrm{ct}}(x) & =\frac{\Sigma^{2}}{2} \frac{I_{2}(2 z)}{z I_{1}(2 z)},
\end{aligned}
$$

where $z$ is defined in (4.7). Hence, for the PCAC mass we find the expected result

$$
m_{\mathrm{PCAC}}^{\mathrm{LO}}=m_{\mathrm{P}} \cos \omega_{0}=m .
$$

Defining maximal twist by a vanishing PCAC mass is therefore equivalent to $m=0$, at least to this order in the chiral expansion.

The leading correction in the $\mathrm{GSM}^{*}$ regime is given by (4.1). The NLO result for the PCAC mass is then found to be

$$
\begin{aligned}
m_{\mathrm{PCAC}}^{\mathrm{NLO}} & =m_{\mathrm{P}} \cos \omega_{0}\left(1+\rho \Delta_{m}\right)=m\left(1+\rho \Delta_{m}\right), \\
\Delta_{m} & =\frac{2}{z^{2}}-\frac{I_{1}(2 z)}{z I_{2}(2 z)} .
\end{aligned}
$$

The main observation we can make is that the PCAC mass is still proportional to $m$. Consequently, maximal twist, given by a vanishing PCAC mass, is still equivalent to $m=0$. This is perhaps better seen if we reformulate (4.16) in terms of the twist angles,

$$
\tan \omega=\left(1-\rho \Delta_{m}\right) \tan \omega_{0} .
$$

This implies that $\omega=\pi / 2$ is equivalent to $\omega_{0}=\pi / 2$ at NLO. This is another way of saying that $m_{\mathrm{PCAC}}^{\mathrm{NLO}}$ vanishes if $m=0$. Note that $\omega=\omega_{0}$ only for angles $\pm \pi / 2$ and 0 . For all other values full use of (4.17) has to be made.

Eq. (4.15) can be inverted to obtain $m$ as a function of $m_{\mathrm{PCAC}}$,

$$
m=m_{\mathrm{PCAC}}\left[1-\rho \Delta_{m}\right],
$$

where in the correction $\Delta_{m}$ we can use the LO result (4.14) and replace $m$ by $m_{\mathrm{PCAC}}$. This is the result we already anticipated in eq. (2.15). In the following we will use it to express correlators as functions of $m_{\mathrm{PCAC}}$ instead of $m$. 


\subsection{Results}

Our results are presented in terms of

$$
z_{m}=m_{\mathrm{PCAC}} \Sigma V, \quad z_{\mu}=\mu \Sigma V .
$$

For $z_{m}$ we keep the same symbol as in eq. (4.5), but we now substitute $m$ with $m_{\text {PCAC }}$ given in eq. (4.18). In order to make our formulae more readable we also use

$$
z=\sqrt{z_{\mu}^{2}+z_{m}^{2}}
$$

\subsubsection{Scalar and pseudoscalar correlators}

It is convenient to write the $\mathrm{O}\left(a^{2}\right)$ correction to the time correlator in eq. (4.10) in the form

$$
C_{P P, a^{2}}^{a b}=\rho \frac{L^{3} \Sigma^{2}}{2} \Delta_{P P}^{a b}, \quad C_{S S, a^{2}}^{00}=\rho \frac{L^{3} \Sigma^{2}}{2} \Delta_{S S}^{00} .
$$

For the pseudoscalar correlator we obtain

$$
\begin{aligned}
\Delta_{P P}^{11,22}= & \frac{1}{2 z^{5} I_{1}(2 z)^{2} I_{2}(2 z)}\left\{4 z_{m}{ }^{2}\left(z_{\mu}{ }^{2}+z_{m}{ }^{2}\right) I_{1}(2 z)^{3}+\right. \\
& -z\left(z_{\mu}{ }^{2}+11 z_{m}{ }^{2}\right) I_{2}(2 z) I_{1}(2 z)^{2}+ \\
& +2\left(-2 z_{m}{ }^{4}+3 z_{m}{ }^{2}+z_{\mu}{ }^{2}\left(1-2 z_{m}{ }^{2}\right)\right) I_{2}(2 z)^{2} I_{1}(2 z)+ \\
& \left.+z\left(z_{\mu}{ }^{2}+5 z_{m}{ }^{2}\right) I_{2}(2 z)^{3}\right\}, \\
\Delta_{P P}^{33}= & \frac{1}{2 z^{7} I_{1}(2 z)^{2} I_{2}(2 z)}\left\{-4\left(-z_{m}{ }^{6}+4 z_{\mu}{ }^{2} z_{m}{ }^{4}+5 z_{\mu}{ }^{4} z_{m}{ }^{2}\right) I_{1}(2 z)^{3}+\right. \\
& +z\left(5 z_{\mu}{ }^{4}+42 z_{m}{ }^{2} z_{\mu}{ }^{2}-11 z_{m}{ }^{4}\right) I_{2}(2 z) I_{1}(2 z)^{2}+ \\
& +2\left(-2 z_{m}{ }^{6}+3 z_{m}{ }^{4}+5 z_{\mu}{ }^{4}\left(2 z_{m}{ }^{2}-1\right)+z_{\mu}{ }^{2}\left(8 z_{m}{ }^{4}-2 z_{m}{ }^{2}\right)\right) I_{2}(2 z)^{2} I_{1}(2 z)+ \\
& \left.+z\left(-3 z_{\mu}{ }^{4}-14 z_{m}{ }^{2} z_{\mu}{ }^{2}+5 z_{m}{ }^{4}\right) I_{2}(2 z)^{3}\right\} .
\end{aligned}
$$

The Wilson untwisted case corresponds to $z_{\mu}=0$. In this limit we obtain

$$
\begin{aligned}
\left.\Delta_{P P}^{a b}\right|_{\omega=0}= & \frac{\delta^{a b}}{2 z_{m}^{3} I_{1}\left(2 z_{m}\right)^{2} I_{2}\left(2 z_{m}\right)}\left\{4 z_{m}^{2} I_{1}\left(2 z_{m}\right)^{3}-11 z_{m} I_{2}\left(2 z_{m}\right) I_{1}\left(2 z_{m}\right)^{2}+\right. \\
& \left.+2\left(3-2 z_{m}^{2}\right) I_{2}\left(2 z_{m}\right)^{2} I_{1}\left(2 z_{m}\right)+5 z_{m} I_{2}\left(2 z_{m}\right)^{3}\right\}
\end{aligned}
$$

which reproduces the result reported in [39] (eq. (4.46)) and [38] (eq. (4.4)).

The most interesting case is maximal twist, which is obtained for $z_{m}=0$. In this case the results simplify to

$$
\begin{aligned}
\left.\Delta_{P P}^{11,22}\right|_{\omega=\pi / 2} & =\frac{-z_{\mu} I_{1}\left(2 z_{\mu}\right)^{2}+2 I_{2}\left(2 z_{\mu}\right) I_{1}\left(2 z_{\mu}\right)+z_{\mu} I_{2}\left(2 z_{\mu}\right)^{2}}{2 z_{\mu}^{3} I_{1}\left(2 z_{\mu}\right)^{2}} \\
\left.\Delta_{P P}^{33}\right|_{\omega=\pi / 2} & =\frac{5 z_{\mu} I_{1}\left(2 z_{\mu}\right)^{2}-10 I_{2}\left(2 z_{\mu}\right) I_{1}\left(2 z_{\mu}\right)-3 z_{\mu} I_{2}\left(2 z_{\mu}\right)^{2}}{2 z_{\mu}^{3} I_{1}\left(2 z_{\mu}\right)^{2}} .
\end{aligned}
$$


Notice that both corrections $\Delta_{P P}^{11,22}$ and $\Delta_{P P}^{33}$ are finite in the limit $z_{\mu} \rightarrow 0$.

The $\mathrm{O}\left(a^{2}\right)$ corrections for the scalar singlet and pseudoscalar correlators are related by

$$
\frac{\Delta_{S S}^{00}}{4}+\sum_{a=1}^{3} \Delta_{P P}^{a a}=0,
$$

which is valid for generic twist angle, at least to this order in the chiral expansion. This result leads to a generalization of what has already been found for untwisted masses [38]: The combination

$$
\frac{C_{S S}^{00}(t)}{4}+\sum_{a=1}^{3} C_{P P}^{a a}(t)
$$

of correlation functions is free from $\mathrm{O}\left(a^{2}\right)$ corrections.

\subsubsection{Axial and vector correlators}

For the $\mathrm{O}\left(a^{2}\right)$ correction in eq. (4.10) to the time-component axial and vector correlators we find

$$
C_{X Y, a^{2}}^{a b}=-\rho \frac{F^{2}}{2 T} \Delta_{X Y}^{a b}, \quad X, Y=A, V,
$$

where $\Delta_{A A}^{a b}$ is given by

$$
\begin{aligned}
\Delta_{A A}^{11,22}= & \frac{1}{z^{7} I_{1}(2 z)^{2} I_{2}(2 z)}\left\{4\left(-z_{m}{ }^{6}+z_{\mu}{ }^{2} z_{m}{ }^{4}+2 z_{\mu}{ }^{4} z_{m}{ }^{2}\right) I_{1}(2 z)^{3}+\right. \\
& +z\left(-2 z_{\mu}{ }^{4}-15 z_{m}{ }^{2} z_{\mu}{ }^{2}+11 z_{m}{ }^{4}\right) I_{2}(2 z) I_{1}(2 z)^{2}+ \\
& -2\left(-2 z_{m}{ }^{6}+3 z_{m}{ }^{4}+z_{\mu}{ }^{4}\left(4 z_{m}{ }^{2}-2\right)+z_{\mu}{ }^{2}\left(2 z_{m}{ }^{4}+z_{m}{ }^{2}\right)\right) I_{2}(2 z)^{2} I_{1}(2 z)+ \\
& \left.+z\left(z_{\mu}{ }^{4}+4 z_{m}{ }^{2} z_{\mu}{ }^{2}-5 z_{m}{ }^{4}\right) I_{2}(2 z)^{3}\right\} \\
\Delta_{A A}^{33}= & \frac{1}{z^{5} I_{1}(2 z)^{2} I_{2}(2 z)}\left\{-4 z_{m}{ }^{2} z^{2} I_{1}(2 z)^{3}+z\left(z_{\mu}{ }^{2}+11 z_{m}{ }^{2}\right) I_{2}(2 z) I_{1}(2 z)^{2}+\right. \\
& \left.+2\left(\left(2 z_{m}{ }^{2}-1\right) z_{\mu}{ }^{2}+z_{m}{ }^{2}\left(2 z_{m}{ }^{2}-3\right)\right) I_{2}(2 z)^{2} I_{1}(2 z)-z\left(z_{\mu}{ }^{2}+5 z_{m}{ }^{2}\right) I_{2}(2 z)^{3}\right\} .
\end{aligned}
$$

Also in this case one can verify that for $z_{\mu}=0$ one obtains the Wilson untwisted formula [39]

$$
\left.\Delta_{A A}^{a b}\right|_{\omega=0}=-\left.2 \Delta_{P P}^{a b}\right|_{\omega=0} .
$$

At maximal twist we obtain

$$
\begin{aligned}
\Delta_{A A, \omega=\pi / 2}^{11,22} & =\frac{-2 z_{\mu} I_{1}\left(2 z_{\mu}\right)^{2}+4 I_{2}\left(2 z_{\mu}\right) I_{1}\left(2 z_{\mu}\right)+z_{\mu} I_{2}\left(2 z_{\mu}\right)^{2}}{z_{\mu}^{3} I_{1}\left(2 z_{\mu}\right)^{2}}, \\
\Delta_{A A, \omega=\pi / 2}^{33} & =\frac{z_{\mu} I_{1}\left(2 z_{\mu}\right)^{2}-2 I_{2}\left(2 z_{\mu}\right) I_{1}\left(2 z_{\mu}\right)-z_{\mu} I_{2}\left(2 z_{\mu}\right)^{2}}{z_{\mu}^{3} I_{1}\left(2 z_{\mu}\right)^{2}} .
\end{aligned}
$$


The $\mathrm{O}\left(a^{2}\right)$ correction for the vector correlator is, up to a sign, the same as for the axial vector correlator,

$$
\Delta_{V V, a^{2}}^{a b}=-\Delta_{A A, a^{2}}^{a b}
$$

This identity holds for generic twist angles and generalizes the result for untwisted masses [39]. An obvious consequence is that for correlation functions of right- and lefthanded currents $J_{\mu, L, R}^{a}=\frac{1}{2}\left(V_{\mu}^{a} \pm A_{\mu}^{a}\right)$ the leading $\mathrm{O}\left(a^{2}\right)$ corrections cancel. This follows from the fact that these currents do not contain zero modes at LO, hence the connected and disconnected contributions in eq. (4.1) cancel among each others.

\subsection{Numerical estimates}

For the correlators considered, the leading $\mathrm{O}\left(a^{2}\right)$ correction in the $\mathrm{GSM}^{*}$ regime is just a shift of the constant part. In order to get estimates for the size of these corrections in typical present-day simulations we look at the ratios

$$
R_{X Y}^{a b}=\left|\frac{C_{X Y, a^{2}}^{a b}(T / 2)}{C_{X Y, \mathrm{ct}}^{a b}(T / 2)}\right| .
$$

These ratios are the relative shift of the correlators at the midpoint $t=T / 2$.

Approximate values for the parameters entering the correlators are taken from the simulations of the ETM collaboration $[26,59]$. We use $F=90 \mathrm{MeV}$ and $a=0.063 \mathrm{fm}$. For simplicity we assume a hypercubic lattice with $N_{T}=N_{L}=24$, which corresponds to a box size $L=1.512 \mathrm{fm}$. This is rather small and the $\epsilon$-expansion might not converge well, but here we are interested only in an estimate about the order of magnitude for the lattice spacing corrections.

The coefficient $c_{2}$ is estimated from the pion mass splitting together with the LO ChPT prediction $-2 c_{2} a^{2}=m_{\pi^{ \pm}}^{2}-m_{\pi^{0}}^{2}$ [36]. The data for the charged and neutral pion masses in ref. [59] translates into $\left|c_{2}\right| \approx(600 \mathrm{MeV})^{4}$. The error, however, is quite large because of the large statistical uncertainty in the determination of the neutral pion mass. ${ }^{8}$ $\left|c_{2}\right| \approx(600 \mathrm{MeV})^{4}$ implies $\rho \approx 0.75$. Although this is slightly large it is smaller than 1 and we may still count this as $\mathrm{O}\left(\epsilon^{2}\right)$, as we should in the GSM* regime.

Figure 1 shows $R_{P P}^{11,22}, R_{P P}^{33}, R_{V V}^{11,22}$ and $R_{V V}^{33}$ for maximal twist $\left(z_{m}=0\right)$ and $z=z_{\mu}$ values in the $\epsilon$-regime. For $z_{\mu}=1.0$ we find values less than 2.5 percent, decreasing to less than 2 percent for $z_{\mu}=2.5$. The ratios for flavor index $a=b=1,2$ and $a=b=3$ assume the same value for vanishing $z_{\mu}$, since for vanishing twisted mass we restore isospin symmetry. At $z_{\mu}=0$ the corrections are maximal but for small values $z_{\mu}$ we eventually enter the Aoki regime and our formulae cease to be valid.

The curves in figure 1 look qualitatively very similar to the ones for untwisted masses shown in ref. [39], although the decrease of the ratios for growing $z_{\mu}$ is slightly faster in the untwisted case.

\footnotetext{
${ }^{8}$ Note that the value for $c_{2}$ is not universal but depends on all the details of the lattice action chosen in the simulation. An analysis [60] of quenched twisted mass lattice data led to the value $c_{2} \approx(300 \mathrm{MeV})^{4}$.
} 


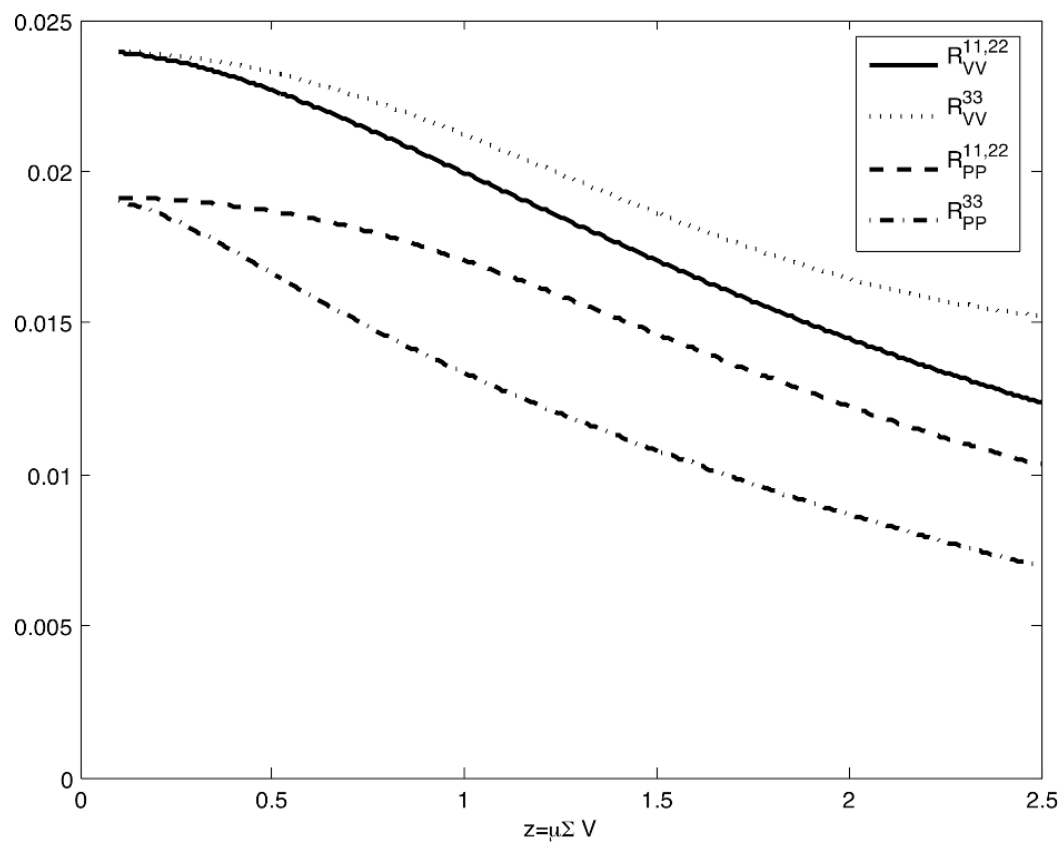

Figure 1. Ratios for the pseudoscalar and vector current correlators as a function of $z=z_{\mu}=\mu \Sigma V$.

Figure 2 shows the ratios involving the axial vector current, $R_{A A}^{11,22}$ and $R_{A A}^{33}$. The ratio $R_{A A}^{33}$ shows a behavior similar to the ratios plotted in figure 1 . In contrast, $R_{A A}^{11,22}$ increases with increasing $z_{\mu}$, up to about 6 percent for $z_{\mu}=2.5$. The reason for this somewhat odd feature is not that the $\mathrm{O}\left(a^{2}\right)$ corrections are larger for this particular correlator. The origin for the increase in $R_{A A}^{11,22}$ is the continuum correlator in the denominator of the ratio. $C_{A A, \text { ct }}^{11,22}$ deceases much more rapidly with increasing $z_{\mu}$ than $C_{A A, a^{2}}^{11,22}$ in the numerator, leading to an increasing ratio $R_{A A}^{11,22}$. In fact, the result for $R_{A A}^{11,22}$ will eventually diverge for large $z_{\mu}$ where the continuum correlator has a zero. Notice that this happens in a region where $z \gg 1$ (at fixed volume), which is not expected to be in the domain of validity of the $\epsilon$-expansion. Figure 3 shows directly the correlators $C_{A A}^{11,22}(T / 2)$ and $C_{A A, \mathrm{ct}}^{11,22}(T / 2)$, both divided by $\left(-F^{2} / T\right)$ in order to get dimensionless quantities. Obviously, the correlators are well behaved and $\mathrm{O}\left(a^{2}\right)$ correction gives a small and almost constant shift of the continuum result.

We conclude that, for our choice of parameters, the $\mathrm{O}\left(a^{2}\right)$ corrections to the correlators are at the few percent level, a small and probably negligible correction.

\section{Concluding remarks}

We have extended the framework of the $N_{f}=2$ Wilson chiral effective theory in the $\epsilon$ regime to the case of a twisted mass. By keeping the same power counting for the untwisted mass $m$ and for the twisted mass $\mu$, we have defined different regimes along the same lines as in the pure Wilson case $[38,39]$. For quark masses of the order $a \Lambda_{\mathrm{QCD}}^{2}$ (GSM regime), the explicit breaking of chiral symmetry induced by the lattice spacing is strongly suppressed, and lattice artefacts appear only at NNLO in the epsilon expansion. On the other hand, if 


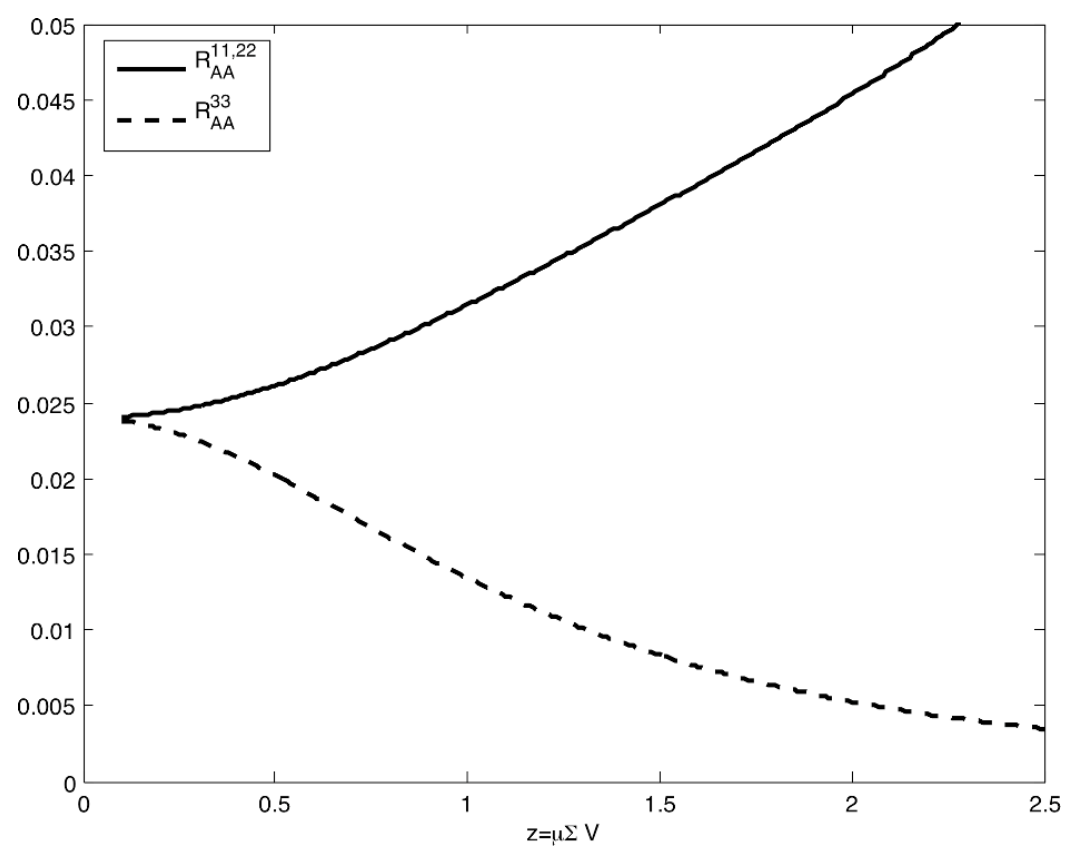

Figure 2. Ratios for the axial vector correlators as a function of $z=z_{\mu}=\mu \Sigma V$.

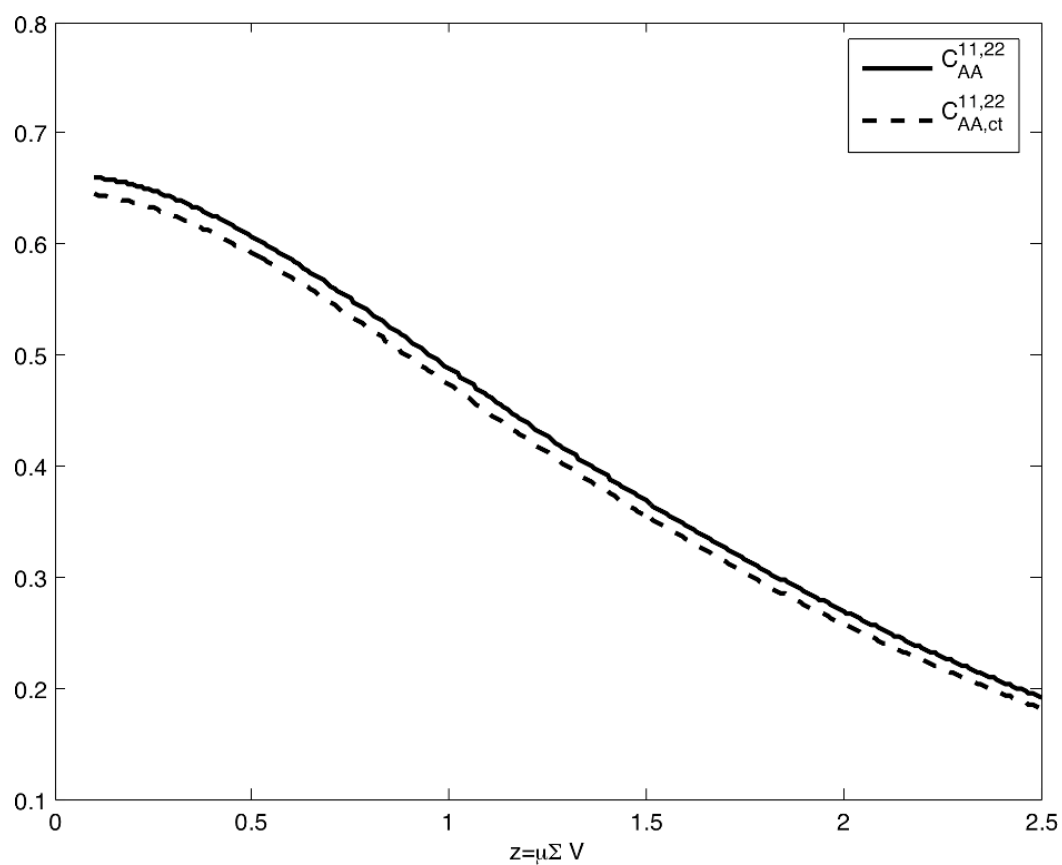

Figure 3. The axial vector correlator with $a=1,2$ (normalized by $-F^{2} / T$ ) as a function of $z=z_{\mu}=\mu \Sigma V$.

the quark masses are of order $a^{2} \Lambda_{\mathrm{QCD}}^{3}$ (Aoki regime), discretization effects appear already at LO. In this paper we have focused on the intermediate $\left(\mathrm{GSM}^{*}\right)$ regime, where lattice artefacts start to contribute at NLO, which is the order at which the matching between lattice data and the chiral effective theory is usually performed. We have computed those 
leading effects for several mesonic two-point functions (pseudoscalar, scalar singlet, axial and vector). The interesting feature of this regime is that only the $\mathrm{O}\left(a^{2}\right)$ corrections in the chiral Lagrangian contribute. There is no proliferation of unknown couplings: apart from the continuum leading order couplings $\Sigma$ and $F$, only an extra constant $c_{2}$ appears.

We have computed the leading $\mathrm{O}\left(a^{2}\right)$ corrections to the PCAC quark mass and expressed the correlators as a function of the dimensionless variables $z_{m}=m_{\mathrm{PCAC}} \Sigma V$ and $z_{\mu}=\mu \Sigma V$. We have adopted the so-called twisted basis, where isospin breaking for nonzero twist angle explicitly shows up.

The final formulae we quote are valid for an arbitrary twist angle, and hence reproduce also the untwisted Wilson case considered in [38, 39], for $\omega=0$ (equivalent to $\mu=0$ ). A particularly interesting setup is maximal twist $\omega=\pi / 2$, here defined by $m_{\mathrm{PCAC}}=0$, where automatic $\mathrm{O}(a)$ improvement occurs [50]. The numerical investigations performed in section 4.4 suggest that, like for untwisted Wilson fermions, for typical lattice parameters adopted in present-day simulations, the $\mathrm{O}\left(a^{2}\right)$ corrections remain at the few percent level. This result supports the possibility to extract low-energy couplings with twisted mass Wilson fermions simulations in the $\epsilon$-regime with controlled systematic errors. Notice, however, that it is not possible to predict a priori in which particular regime (GSM, Aoki) one actually has performed a simulation, and a scaling study is advocated.

We finally remark that a determination of the twist angle in the $\epsilon$-regime might be numerically not so easy. To determine the actual value of $\omega$ with a reasonable accuracy the statistical and systematic uncertainties on the PCAC mass have to be ideally much smaller than the value of the twisted mass $\mu$. In the $\epsilon$-regime, where the value of the twisted mass could become comparable to the errors associated with the PCAC mass, this task could become extremely difficult. This fact could induce large uncertainties in the determination of the twist angle. A realistic procedure is to determine the twist angle from $p$-regime simulations $[59,61]$ where it is possible to keep the uncertainty on the twist angle well under control. The bare parameters tuned in the $p$-regime are then used in the $\epsilon$ regime. We expect that this choice will induce $\mathrm{O}(a)$ corrections to the PCAC mass, which will not spoil automatic $\mathrm{O}(a)$ improvement and the validity of the formulae given in this paper. An alternative procedure is to use correlation functions which are $\omega$ independent at the classical level. As we have seen in section 4.3 (cf. eqs. (4.27) and (4.35)) these particular linear combinations are $\omega$ independent also at NLO in the GSM* regime and additionally they are free from $\mathrm{O}\left(a^{2}\right)$ corrections. This is an alternative procedure to analyze lattice data which is free from the uncertainties stemming from the determination of the twist angle and free from the $\mathrm{O}\left(a^{2}\right)$ corrections affecting the standard correlation functions.

\section{Acknowledgments}

This work is partially supported by EC Sixth Framework Program under the contract MRTN-CT-2006-035482 (FLAVIAnet) and by the Deutsche Forschungsgemeinschaft (SFB/TR 09). A.S. acknowledges discussions with K. Jansen and C. Michael. A.S. would like to thank for the pleasant and stimulating atmosphere all the members of the Theoretical Division of the University of Liverpool, where a big part of this work has been done. 
A.S. also acknowledges financial support from Spanish Consolider-Ingenio 2010 Programme CPAN (CSD 2007-00042) and from Comunidad Autónoma de Madrid, CAM under grant HEPHACOS P-ESP-00346.

\section{A Selected formulae and definitions for the epsilon regime}

In this appendix we briefly summarize formulae which are relevant for the computation of correlation functions in the $\epsilon$-regime of chiral perturbation theory. For more details the reader can refer to $[38,39]$. The pseudo Nambu-Goldstone bosons propagators for the nonzero modes is defined as

$$
\bar{G}(x)=\frac{1}{V} \sum_{p \neq 0} \frac{e^{i p x}}{p^{2}} .
$$

Following refs. $[52,54]$ we define

$$
\begin{aligned}
\bar{G}(0) & \equiv-\frac{\beta_{1}}{\sqrt{V}}, \\
T \frac{d}{d T} \bar{G}(0) & \equiv \frac{T^{2} k_{00}}{V},
\end{aligned}
$$

where $\beta_{1}$ and $k_{00}$ are finite dimensionless shape coefficients which depend on the geometry and can be evaluated numerically.

The integral over spatial components of the propagators $\bar{G}(x)$ yields the parabolic function $h_{1}(t / T)$ :

$$
\int d^{3} \vec{x} \bar{G}(x)=T h_{1}\left(\frac{t}{T}\right)=\frac{T}{2}\left[\left(\left|\frac{t}{T}\right|-\frac{1}{2}\right)^{2}-\frac{1}{12}\right] .
$$

Another quantity which appears frequently is the quark condensate at one loop, which for $N_{f}=2$ is given by [52]

$$
\Sigma_{\text {eff }}=\Sigma\left(1+\frac{3}{2 F^{2}} \frac{\beta_{1}}{\sqrt{V}}\right) .
$$

In the calculation of the NLO continuum correlators the measure factor in eq. (3.10) is needed at $O\left(\epsilon^{2}\right)[4,52]$ :

$$
B(\xi)=\frac{4}{3 F^{2} V} \frac{1}{2} \int d^{4} x \operatorname{Tr}\left(\xi^{a} T^{a} \xi^{b} T^{b}\right) .
$$

\section{B Continuum correlators in the twisted basis}

In this appendix we summarize continuum formulæ for two-point functions in the twisted basis. For simplicity we introduce the function

$$
L(x)=\frac{I_{2}(2 x)}{x I_{1}(2 x)} .
$$

The results are given in terms of the variables

$$
z_{\mu}=\mu \Sigma V, \quad z_{m}=m_{\mathrm{PCAC}} \Sigma V, \quad z=\sqrt{z_{\mu}^{2}+z_{m}^{2}} .
$$


The pure Wilson case corresponds to $z_{\mu}=0, z=z_{m}$, while the maximal twist is verified for $z_{m}=0, z=z_{\mu}$. Each correlator consists of a constant part and on a time-dependent contribution; the time-dependence is represented by the function $h_{1}$ defined in eq. (A.4).

\section{B.1 Scalar and pseudoscalar correlators}

We write the continuum scalar singlet correlator as

$$
C_{S S, c t}^{00}(t)=L^{3} C_{S}^{00}+\alpha_{S}^{00} T h_{1}(t / T)
$$

while for the pseudoscalar correlator we have

$$
C_{P P, c t}^{a b}(t)=L^{3} C_{P}^{a b}+\alpha_{P}^{a b} T h_{1}(t / T) .
$$

The coefficients $C_{S^{0}}, \alpha_{S^{0}}, C_{P}^{a b}$ and $\alpha_{P}^{a b}$ are given by

$$
\begin{aligned}
C_{S}^{00} & =4 \Sigma_{\text {eff }}^{2}\left[\frac{z_{m}^{2}}{z^{2}}-\frac{L\left(z_{\mathrm{eff}}\right)}{2}\left(1-\frac{2\left(z_{\mu}^{2}-z_{m}^{2}\right)}{z^{2}}\right)\right], \\
\alpha_{S}^{00} & =4 \frac{\Sigma^{2}}{F^{2}}\left[\frac{z_{\mu}^{2}}{z^{2}}+\frac{L(z)}{2}\left(1-\frac{2\left(z_{\mu}^{2}-z_{m}^{2}\right)}{z^{2}}\right)\right], \\
C_{P}^{11,22} & =\Sigma_{\text {eff }}^{2} \frac{L\left(z_{\mathrm{eff}}\right)}{2} \\
C_{P}^{33} & =\Sigma_{\text {eff }}^{2}\left[\frac{z_{\mu}^{2}}{z^{2}}-\frac{L\left(z_{\mathrm{eff}}\right)}{2}\left(1+\frac{2\left(z_{\mu}^{2}-z_{m}^{2}\right)}{z^{2}}\right)\right], \\
\alpha_{P}^{11,22} & =\frac{\Sigma^{2}}{F^{2}}\left[1-\frac{L(z)}{2}\right], \\
\alpha_{P}^{33} & =\frac{\Sigma^{2}}{F^{2}}\left[\frac{z_{m}^{2}}{z^{2}}+\frac{L(z)}{2}\left(1+\frac{2\left(z_{\mu}^{2}-z_{m}^{2}\right)}{z^{2}}\right)\right] .
\end{aligned}
$$

The subscript "eff" in $z_{\text {eff }}$ indicates that $\Sigma$ must be substituted by the one-loop corrected quark condensate $\Sigma_{\text {eff }}$ given in eq. (A.5).

\section{B.2 Axial and vector correlators}

The continuum vector and axial correlators are given by

$$
C_{A A, V V, c t}^{a b}(x-y)=-\frac{\alpha_{A, V}^{a b}}{T}+\frac{T}{V} \beta_{A, V}^{a b} k_{00}-\frac{T}{V} \gamma_{A, V}^{a b} h_{1}(t / T) .
$$

Here $\beta_{1}$ and $k_{00}$ are familiar shape factors which depend on the geometry of the space-time volume. They are defined in eqs.(A.2), (A.3). 
Explicit results for the coefficients are:

$$
\begin{aligned}
\alpha_{A}^{11,22} & =F^{2}\left(\frac{z_{m}^{2}}{z^{2}}-\frac{z_{m}^{2}-z_{\mu}^{2}}{z^{2}} L\left(z_{\mathrm{eff}}\right)\right)+\frac{2 \beta_{1}}{\sqrt{V}}\left(\frac{z_{m}^{2}}{z^{2}}-\frac{z_{m}^{2}-z_{\mu}^{2}}{z^{2}} L(z)\right) \\
\alpha_{A}^{33} & =F^{2}\left(1-L\left(z_{\mathrm{eff}}\right)\right)+\frac{2 \beta_{1}}{\sqrt{V}}(1-L(z)) \\
\beta_{A}^{11,22} & =2\left(\frac{z_{\mu}^{2}}{z^{2}}+\frac{z_{m}^{2}-z_{\mu}^{2}}{z^{2}} L(z)\right) \\
\beta_{A}^{33} & =2 L(z) \\
\gamma_{A}^{11,22} & =2 z_{m}^{2} L(z) \\
\gamma_{A}^{33} & =2 z^{2} L(z), \\
\alpha_{V}^{11,22} & =F^{2}\left(\frac{z_{\mu}^{2}}{z^{2}}+\frac{z_{m}^{2}-z_{\mu}^{2}}{z^{2}} L\left(z_{\mathrm{eff}}\right)\right)+\frac{2 \beta_{1}}{\sqrt{V}}\left(\frac{z_{\mu}^{2}}{z^{2}}+\frac{z_{m}^{2}-z_{\mu}^{2}}{z^{2}} L(z)\right) \\
\alpha_{V}^{33} & =F^{2} L\left(z_{\mathrm{eff}}\right)+\frac{2 \beta_{1}}{\sqrt{V}} L(z) \\
\beta_{V}^{11,22} & =2\left(\frac{z_{m}^{2}}{z^{2}}-\frac{z_{m}^{2}-z_{\mu}^{2}}{z^{2}} L(z)\right) \\
\beta_{V}^{33} & =2(1-L(z)), \\
\gamma_{V}^{11,22} & =2 z_{\mu}^{2} L(z), \\
\gamma_{V}^{33} & =0
\end{aligned}
$$

Also in this case, the subscript "eff" in $z_{\text {eff }}$ indicates that $\Sigma$ must be substituted by the one-loop corrected quark condensate $\Sigma_{\text {eff }}$ given in eq. (A.5).

\section{Notations for comparison with ref. [38]}

In ref. [38] a slightly different notation has been used in comparison with this work. Here we give a short summary of the main differences. In ref. [38] the variables $z$ and $z_{2}$ have been introduced. They correspond respectively to $z_{m} / 2$ (cf. eq. (4.5)) and $-\rho$ (cf. eq. (4.3)).

In the following for the $z$ variables we will use the same notation adopted in the main text given in eqs. (4.19) and (4.20). The Bessel functions $I_{n}(x)$ translate to the function $X_{n}(x)$ used in [38] as

$$
I_{n}=\frac{(x / 2)^{n}}{\sqrt{\pi} \Gamma(n+1 / 2)} X_{n} .
$$

As examples we give here few ratios which typically appear in correlation functions

$$
\frac{I_{2}}{I_{1}}=\frac{2 z}{3} \frac{X_{2}}{X_{1}}=\frac{X_{1}^{\prime}}{X_{1}}, \quad \frac{I_{3}}{I_{1}}=\frac{4 z^{2}}{15} \frac{X_{2}}{X_{1}},
$$

To conclude we find it useful to write the following correlation function

$$
C_{P P}^{11}(t)=C_{P P, \mathrm{ct}}^{11}(t)+C_{P P, a^{2}}^{11} .
$$


with the notations of ref. [38]. We have

$$
C_{P P, \mathrm{ct}}^{11}(t)=\frac{\Sigma_{\text {eff }}^{2}}{3}\left\{\frac{X_{2}}{X_{1}}+\frac{3}{F^{2}}\left[1-\frac{1}{3} \frac{X_{2}}{X_{1}}\right] \frac{T}{L^{3}} h_{1}(t / T)\right\}
$$

and

$$
\begin{aligned}
C_{P P, a^{2}}^{11}= & \frac{z_{2} L^{3} \Sigma^{2}}{3}\left\{48 \frac{z_{m}^{2}}{z^{4}}-\left(64 \frac{z_{m}^{2}}{z^{4}}-2 \frac{z_{m}^{2}}{z^{2}}\right)\left(\frac{X_{2}}{X_{1}}\right)-\left(\frac{32}{3 z^{2}}+\frac{z_{m}^{2}}{z^{2}}-\frac{z_{\mu}^{2}}{3 z^{2}}\right)\left(\frac{X_{2}}{X_{1}}\right)^{2}\right. \\
& \left.-9 \frac{z_{m}^{2}}{z^{4}}\left(\frac{X_{1}}{X_{2}}\right)-\left(\frac{z_{m}^{2}}{z^{2}}-\frac{z_{\mu}}{5 z^{2}}\right)\left(\frac{X_{3}}{X_{1}}\right)\right\}
\end{aligned}
$$

where $X_{n}=X_{n}(2 z)$ with $z$ defined in eq. (4.20).

\section{Example of group integrals with isospin breaking}

In this appendix we show one of the procedures we have used to perform the zero-modes integrals with isospin breaking integrands. We use the example of calculating the expectation value $\left\langle\delta S_{a^{2}}\right\rangle$, which is part of the correction (4.1) we are interested in. To simplify the calculation we restrict ourselves to the case of maximal twist with $m=0$, which nevertheless demonstrates the main idea. In this case the integral we have to perform reads (c.f. eqs. (4.2) and (4.4))

$$
\left\langle\delta S_{a^{2}}\right\rangle=\frac{\rho}{16 Z_{0}} \int \mathrm{d}\left[U_{0}\right]\left(\operatorname{Tr}\left(U_{0}+U_{0}^{\dagger}\right)\right)^{2} e^{-i \frac{z \mu}{2} \operatorname{Tr}\left[\sigma^{3}\left(U_{0}-U_{0}^{\dagger}\right)\right]} .
$$

Performing the change of variables $U_{0} \rightarrow i \sigma^{3} U_{0}$ (which is equivalent to the rotation (2.5) with $\left.\omega_{0}=\pi / 2\right)$ this translates into

$$
\left\langle\delta S_{a^{2}}\right\rangle=-\frac{\rho}{16 Z_{0}} \int \mathrm{d}\left[U_{0}\right]\left(\operatorname{Tr}\left(\sigma^{3}\left[U_{0}-U_{0}^{\dagger}\right]\right)\right)^{2} e^{\frac{z_{\mu}}{2} \operatorname{Tr}\left[U_{0}+U_{0}^{\dagger}\right]} .
$$

If we use the parametrization

$$
U_{0}=\exp [i \phi \vec{n} \cdot \vec{\sigma} / 2]
$$

$(\phi \in[0,2 \pi],|\vec{n}|=1)$ for the constant mode, the Haar measure reads

$$
\mathrm{d}\left[U_{0}\right]=\frac{1}{4 \pi^{2}} \mathrm{~d} \phi \mathrm{d} \Omega \sin ^{2} \frac{\phi}{2},
$$

with $\mathrm{d} \Omega=\mathrm{d} \Omega(\vec{n})$ being the measure of the 2-sphere, and the integral turns into

$$
\left\langle\delta S_{a^{2}}\right\rangle=\rho \frac{1}{Z_{0}} \int \mathrm{d}\left[U_{0}\right] e^{2 z_{\mu} \cos \phi} \sin ^{2} \frac{\phi}{2} n_{3}^{2} .
$$

The factor $n_{3}^{2}$ in the integrand is a remnant of isospin breaking. Without isospin breaking the integrals one faces involve functions of $\phi$ only, at least if various completeness relations for the group generators are used [52]. In this case the integration $\int \mathrm{d} \Omega$ gives a trivial 
factor $4 \pi$ and the remaining integral over $\phi$ leads to expressions involving modified Bessel functions. In order to make contact to these known integrals we write

$$
\int_{S^{2}} \mathrm{~d} \Omega n_{3}^{2}=\frac{1}{3} \int_{S^{2}} \mathrm{~d} \Omega
$$

and the integral we are interested in turns into

$$
\left\langle\delta S_{a^{2}}\right\rangle=\frac{\rho}{3} \frac{1}{Z_{0}} \int \mathrm{d}\left[U_{0}\right] e^{2 z_{\mu} \cos \phi} \sin ^{2} \frac{\phi}{2} .
$$

For the remaining integration over $\phi$ we write $\sin ^{2}(\phi / 2)=1-\cos ^{2}(\phi / 2)=1-\left(\operatorname{Tr} U_{0}\right)^{2} / 4$, and we finally obtain

$$
\left\langle\delta S_{a^{2}}\right\rangle=\frac{\rho}{3}\left[\langle 1\rangle_{\mathrm{phys}}-\frac{1}{4}\left\langle\left(\operatorname{Tr} U_{0}\right)^{2}\right\rangle_{\mathrm{phys}}\right] .
$$

This is our desired result: The right hand side involves familiar integrals with the standard Boltzmann weight, c.f. (4.6) (as indicated by the subscript "phys"). A useful collection of relevant integrals is given in appendix B of ref. [39], which can be used to express (D.8) as

$$
\left\langle\delta S_{a^{2}}\right\rangle=\rho \frac{1}{2 z_{\mu}} \frac{I_{2}\left(2 z_{\mu}\right)}{I_{1}\left(2 z_{\mu}\right)} .
$$

The same steps can be carried out in the calculation of correlators $\left\langle O_{1}^{a} O_{2}^{b}\right\rangle$. The integrand will be a product $f(\phi) p\left(n_{3}\right)$, where $f$ is a function of $\phi$ only and $p$ denotes a polynomial in $n_{3}$. Generalizing eq. (D.6), the integration over $S^{2}$ is trivial and gives a simple factor $c_{p}$,

$$
\int_{S^{2}} \mathrm{~d} \Omega p\left(n_{3}\right)=c_{p} \int_{S^{2}} \mathrm{~d} \Omega .
$$

The remaining integral can be expressed in terms of familiar integrals known from continuum $\epsilon$-regime calculations without isospin breaking.

Open Access. This article is distributed under the terms of the Creative Commons Attribution Noncommercial License which permits any noncommercial use, distribution, and reproduction in any medium, provided the original author(s) and source are credited.

\section{References}

[1] E.E. Scholz, Light hadron masses and decay constants, arXiv:0911.2191 [SPIRES].

[2] C. Jung, Status of dynamical ensemble generation, arXiv:1001.0941 [SPIRES].

[3] J. Gasser and H. Leutwyler, Light quarks at low temperatures, Phys. Lett. B 184 (1987) 83 [SPIRES].

[4] J. Gasser and H. Leutwyler, Thermodynamics of chiral symmetry, Phys. Lett. B 188 (1987) 477 [SPIRES].

[5] P. Hernández, K. Jansen and L. Lellouch, Finite-size scaling of the quark condensate in quenched lattice QCD, Phys. Lett. B 469 (1999) 198 [hep-lat/9907022] [SPIRES]. 
[6] MILC collaboration, T.A. DeGrand, Another determination of the quark condensate from an overlap action, Phys. Rev. D 64 (2001) 117501 [hep-lat/0107014] [SPIRES].

[7] P. Hasenfratz, S. Hauswirth, T. Jörg, F. Niedermayer and K. Holland, Testing the fixed-point QCD action and the construction of chiral currents, Nucl. Phys. B 643 (2002) 280 [hep-lat/0205010] [SPIRES].

[8] W. Bietenholz, T. Chiarappa, K. Jansen, K.I. Nagai and S. Shcheredin, Axial correlation functions in the $\epsilon$-regime: a numerical study with overlap fermions, JHEP 02 (2004) 023 [hep-lat/0311012] [SPIRES].

[9] L. Giusti, P. Hernández, M. Laine, P. Weisz and H. Wittig, Low-energy couplings of QCD from current correlators near the chiral limit, JHEP 04 (2004) 013 [hep-lat/0402002] [SPIRES].

[10] H. Fukaya, S. Hashimoto and K. Ogawa, Low-lying mode contribution to the quenched meson correlators in the $\epsilon$-regime, Prog. Theor. Phys. 114 (2005) 451 [hep-lat/0504018] [SPIRES].

[11] W. Bietenholz, T. Chiarappa, K. Jansen, K.I. Nagai and S. Shcheredin, Simulating chiral quarks in the $\epsilon$-regime of QCD, Nucl. Phys. A 755 (2005) 641 [hep-lat/0501012] [SPIRES].

[12] L. Giusti and S. Necco, Spontaneous chiral symmetry breaking in QCD: a finite-size scaling study on the lattice, JHEP 04 (2007) 090 [hep-lat/0702013] [SPIRES].

[13] L. Giusti et al., Testing chiral effective theory with quenched lattice QCD, JHEP 05 (2008) 024 [arXiv:0803.2772] [SPIRES].

[14] P.H. Ginsparg and K.G. Wilson, A remnant of chiral symmetry on the lattice, Phys. Rev. D 25 (1982) 2649 [SPIRES].

[15] T. DeGrand, Z. Liu and S. Schaefer, Quark condensate in two-flavor QCD, Phys. Rev. D 74 (2006) 094504 [Erratum ibid. D 74 (2006) 099904] [hep-lat/0608019] [SPIRES].

[16] C.B. Lang, P. Majumdar and W. Ortner, The condensate for two dynamical chirally improved quarks in QCD, Phys. Lett. B 649 (2007) 225 [hep-lat/0611010] [SPIRES].

[17] T. DeGrand and S. Schaefer, Parameters of the lowest order chiral Lagrangian from fermion eigenvalues, Phys. Rev. D 76 (2007) 094509 [arXiv:0708.1731] [SPIRES].

[18] JLQCD collaboration, H. Fukaya et al., Two-flavor lattice QCD simulation in the $\epsilon$-regime with exact chiral symmetry, Phys. Rev. Lett. 98 (2007) 172001 [hep-lat/0702003] [SPIRES].

[19] H. Fukaya et al., Two-flavor lattice QCD in the $\epsilon$-regime and chiral Random Matrix theory, Phys. Rev. D 76 (2007) 054503 [arXiv:0705.3322] [SPIRES].

[20] JLQCD collaboration, H. Fukaya et al., Lattice study of meson correlators in the $\epsilon$-regime of two-flavor QCD, Phys. Rev. D 77 (2008) 074503 [arXiv:0711.4965] [SPIRES].

[21] P. Hasenfratz et al., 2+1 flavor QCD simulated in the $\epsilon$-regime in different topological sectors, JHEP 11 (2009) 100 [arXiv:0707.0071] [SPIRES].

[22] JLQCD collaboration, H. Fukaya et al., Determination of the chiral condensate from 2+1-flavor lattice QCD, Phys. Rev. Lett. 104 (2010) 122002 [arXiv:0911.5555] [SPIRES].

[23] M. Lüscher, Exact chiral symmetry on the lattice and the Ginsparg-Wilson relation, Phys. Lett. B 428 (1998) 342 [hep-lat/9802011] [SPIRES].

[24] K. Jansen, A. Nube, A. Shindler, C. Urbach and U. Wenger, Exploring the $\epsilon$-regime with twisted mass fermions, PoS(LATTICE 2007)084 [arXiv:0711.1871] [SPIRES]. 
[25] K. Jansen, A. Nube and A. Shindler, Wilson twisted mass fermions in the $\epsilon$-regime, PoS (LATTICE 2008) 083 [arXiv:0810.0300] [SPIRES].

[26] K. Jansen and A. Shindler, The $\epsilon$-regime of chiral perturbation theory with Wilson-type fermions, arXiv:0911.1931 [SPIRES].

[27] A. Hasenfratz, R. Hoffmann and S. Schaefer, Reweighting towards the chiral limit, Phys. Rev. D 78 (2008) 014515 [arXiv:0805.2369] [SPIRES].

[28] A. Hasenfratz, R. Hoffmann and S. Schaefer, Low energy chiral constants from $\epsilon$-regime simulations with improved Wilson fermions, Phys. Rev. D 78 (2008) 054511 [arXiv: 0806.4586] [SPIRES].

[29] M. Lüscher and F. Palombi, Fluctuations and reweighting of the quark determinant on large lattices, PoS(LATTICE 2008) 049 [arXiv:0810.0946] [SPIRES].

[30] S.R. Sharpe and R.L. Singleton, Jr, Spontaneous flavor and parity breaking with Wilson fermions, Phys. Rev. D 58 (1998) 074501 [hep-lat/9804028] [SPIRES].

[31] G. Rupak and N. Shoresh, Chiral perturbation theory for the Wilson lattice action, Phys. Rev. D 66 (2002) 054503 [hep-lat/0201019] [SPIRES].

[32] G. Münster and C. Schmidt, Chiral perturbation theory for lattice QCD with a twisted mass term, Europhys. Lett. 66 (2004) 652 [hep-lat/0311032] [SPIRES].

[33] L. Scorzato, Pion mass splitting and phase structure in twisted mass QCD, Eur. Phys. J. C 37 (2004) 445 [hep-lat/0407023] [SPIRES].

[34] S.R. Sharpe and J.M.S. Wu, The phase diagram of twisted mass lattice QCD, Phys. Rev. D 70 (2004) 094029 [hep-lat/0407025] [SPIRES].

[35] S. Aoki and O. Bär, Twisted-mass QCD, O(a) improvement and Wilson chiral perturbation theory, Phys. Rev. D 70 (2004) 116011 [hep-lat/0409006] [SPIRES].

[36] S.R. Sharpe and J.M.S. Wu, Twisted mass chiral perturbation theory at next-to-leading order, Phys. Rev. D 71 (2005) 074501 [hep-lat/0411021] [SPIRES].

[37] S. Aoki and O. Bär, Automatic $O(a)$ improvement for twisted-mass $Q C D$ in the presence of spontaneous symmetry breaking, Phys. Rev. D 74 (2006) 034511 [hep-lat/0604018] [SPIRES].

[38] A. Shindler, Observations on the Wilson fermions in the $\epsilon$-regime, Phys. Lett. B 672 (2009) 82 [arXiv: 0812.2251] [SPIRES].

[39] O. Bär, S. Necco and S. Schaefer, The $\epsilon$-regime with Wilson fermions, JHEP 03 (2009) 006 [arXiv: 0812.2403] [SPIRES].

[40] P.H. Damgaard, K. Splittorff and J.J.M. Verbaarschot, Microscopic spectrum of the Wilson dirac operator, arXiv:1001.2937 [SPIRES].

[41] S.R. Sharpe, Applications of chiral perturbation theory to lattice $Q C D$, lectures given at the Nara workshop, October 31 - December 11 (2005), Nara, Japan hep-lat/0607016 [SPIRES].

[42] M. Golterman, Applications of chiral perturbation theory to lattice $Q C D$, lectures given at the 2009 Les Houches Summer School 'Modern perspectives in lattice QCD: Quantum field theory and high performance computing', arXiv:0912.4042 [SPIRES].

[43] Alpha collaboration, R. Frezzotti, P.A. Grassi, S. Sint and P. Weisz, Lattice QCD with a chirally twisted mass term, JHEP 08 (2001) 058 [hep-lat/0101001] [SPIRES]. 
[44] A. Shindler, Twisted mass lattice QCD, Phys. Rept. 461 (2008) 37 [arXiv:0707.4093] [SPIRES].

[45] G. Münster, On the phase structure of twisted mass lattice QCD, JHEP 09 (2004) 035 [hep-lat/0407006] [SPIRES].

[46] O. Bär, G. Rupak and N. Shoresh, Chiral perturbation theory at $O\left(a^{2}\right)$ for lattice $Q C D$, Phys. Rev. D 70 (2004) 034508 [hep-lat/0306021] [SPIRES].

[47] S. Aoki, Chiral perturbation theory with Wilson-type fermions including a effects: $N_{f}=2$ degenerate case, Phys. Rev. D 68 (2003) 054508 [hep-lat/0306027] [SPIRES].

[48] S. Aoki, O. Bär and B. Biedermann, Pion scattering in Wilson ChPT, Phys. Rev. D 78 (2008) 114501 [arXiv:0806.4863] [SPIRES].

[49] S. Aoki, O. Bär and S.R. Sharpe, Vector and axial currents in Wilson chiral perturbation theory, Phys. Rev. D 80 (2009) 014506 [arXiv:0905.0804] [SPIRES].

[50] R. Frezzotti and G.C. Rossi, Chirally improving Wilson fermions. I: O(a) improvement, JHEP 08 (2004) 007 [hep-lat/0306014] [SPIRES].

[51] J. Gasser and H. Leutwyler, Spontaneously broken symmetries: effective Lagrangians at finite volume, Nucl. Phys. B 307 (1988) 763 [SPIRES].

[52] F.C. Hansen, Finite size effects in spontaneously broken $\mathrm{SU}(N) \times \mathrm{SU}(N)$ theories, Nucl. Phys. B 345 (1990) 685 [SPIRES].

[53] F.C. Hansen and H. Leutwyler, Charge correlations and topological susceptibility in QCD, Nucl. Phys. B 350 (1991) 201 [SPIRES].

[54] P. Hasenfratz and H. Leutwyler, Goldstone boson related finite size effects in field theory and critical phenomena with O(N) symmetry, Nucl. Phys. B 343 (1990) 241 [SPIRES].

[55] H. Leutwyler and A.V. Smilga, Spectrum of Dirac operator and role of winding number in QCD, Phys. Rev. D 46 (1992) 5607 [SPIRES].

[56] H. Leutwyler, On the foundations of chiral perturbation theory, Ann. Phys. 235 (1994) 165 [hep-ph/9311274] [SPIRES].

[57] D. Espriu and J. Matias, Renormalization and the equivalence theorem: on-shell scheme, Phys. Rev. D 52 (1995) 6530 [hep-ph/9501279] [SPIRES].

[58] P.H. Damgaard, M.C. Diamantini, P. Hernández and K. Jansen, Finite-size scaling of meson propagators, Nucl. Phys. B 629 (2002) 445 [hep-lat/0112016] [SPIRES].

[59] ETM collaboration, R. Baron et al., Light meson physics from maximally twisted mass lattice $Q C D$, arXiv:0911.5061 [SPIRES].

[60] S. Aoki and O. Bär, WChPT analysis of twisted mass lattice data, Eur. Phys. J. A 31 (2007) 481 [hep-lat/0610085] [SPIRES].

[61] ETM collaboration, P. Boucaud et al., Dynamical twisted mass fermions with light quarks: simulation and analysis details, Comput. Phys. Commun. 179 (2008) 695 [arXiv:0803.0224] [SPIRES]. 